\title{
Panax ginseng extract antagonizes the effect of DKK-1-induced catagen-like changes of hair follicles
}

\author{
YONGHEE LEE ${ }^{1}$, SU NA KIM ${ }^{1}$, YONG DEOG HONG ${ }^{1}$, BYUNG CHEOL PARK $^{2}$ and YONGJOO NA ${ }^{1}$ \\ ${ }^{1}$ Amorepacific R\&D Center, Yongin-si, Gyeonggi-do 446-729; ${ }^{2}$ Department of Dermatology, \\ Dankook Medical College, Cheonan, Chungcheongnam-do 31116, Republic of Korea
}

Received January 3, 2017; Accepted August 11, 2017

DOI: $10.3892 / \mathrm{ijmm} .2017 .3107$

\begin{abstract}
It is well known that Panax ginseng (PG) has various pharmacological effects such as anti-aging and antiinflammation. In a previous study, the authors identified that PG extract induced hair growth by means of a mechanism similar to that of minoxidil. In the present study, the inhibitory effect of PG extract on Dickkopf-1 (DKK-1)-induced catagen-like changes in hair follicles (HFs) was investigated in addition to the underlying mechanism of action. The effects of PG extract on cell proliferation, anti-apoptotic effect, and hair growth were observed using cultured outer root sheath (ORS) keratinocytes and human HFs with or without DKK-1 treatment. The PG extract significantly stimulated proliferation and inhibited apoptosis, respectively, in ORS keratinocytes. PG extract treatment affected the expression of apoptosis-related genes Bcl-2 and Bax. DKK-1 inhibited hair growth, and PG extract dramatically reversed the effect of DKK-1 on ex vivo human hair organ culture. PG extract antagonizes DKK-1induced catagen-like changes, in part, through the regulation of apoptosis-related gene expression in HFs. These findings suggested that PG extract may reduce hair loss despite the presence of DKK-1, a strong catagen inducer via apoptosis.
\end{abstract}

\section{Introduction}

Hair follicles (HFs) are complicated organs composed of multiple layers of epithelia of the outer root sheath (ORS) keratinocytes, the matrix and its derivatives; the inner root sheath and hair shaft; and mesenchymal cells called the dermal papilla (DP) $(1,2)$. The DP, which is surrounded by the

Correspondence to: Dr Yongjoo Na, Amorepacific R\&D Center, 1920 Yonggu-daero, Giheung-gu, Yongin-si, Gyeonggi-do 446-729, Republic of Korea

E-mail: nay@amorepacific.com

Abbreviations: PG extract, Panax ginseng extract; ORS, outer root sheath; HFs, hair follicles; DKK-1, Dickkopf-1

Key words: Panax ginseng, hair growth, outer root sheath, hair follicles, Dickkopf-1, apoptosis dermal sheath and the hair matrix, is considered to be essential to hair induction because of secreted diffusible proteins that regulate the growth and activity of the various cells in the follicle $(3,4)$. The ORS keratinocytes of the HF surround the hair fiber and inner root sheath. The ORS keratinocytes is distinct from other epidermal components, being continuous with the surface epidermis. The ORS keratinocytes consist of several layers of cells that can be identified by their unique ultrastructural properties (1). Hair growth and the cycling of HFs requires reciprocal interactions between the human dermal papilla cells (hDPCs) and ORS keratinocytes (5).

Apoptosis can serve a role in follicular miniaturization, but its association with androgenetic alopecia in males is controversial (6-8). Apoptosis is a complex process regulated by the Bcl-2 gene family (9). The family members act as anti- or pro-apoptotic regulators that are involved in a wide variety of cellular activities. Bcl-2, an apoptosis inhibitor, and Bax, an apoptosis promoter, show tightly regulated, hair cycledependent expression patterns (10). Normal HFs also express high levels of anti-apoptotic protein Bcl-2 (6,7).

Dickkopf-1 (DKK-1), which is a potent antagonist of the Wnt/ $\beta$-catenin signaling pathway, is inducible by dihydrotestosterone (DHT) and promotes catagen progression and the apoptotic cell death of HFs $(11)$. Kwack et al $(12,13)$ demonstrated that DKK-1 is secreted from hDPCs in response to DHT and that it promotes the regression of HFs by blocking Wnt/ $\beta$-catenin signaling and by inhibiting the growth of ORS keratinocytes and triggering apoptotic cell death. The reports also identified that, although DKK-1 treatment rapidly changed the anti-apoptotic protein Bcl-2, DKK-1 promoted the pro-apoptotic protein $\mathrm{Bax}$ in a dose-dependent manner in ORS keratinocytes.

Panax ginseng (PG) has a wide range of pharmacological effects including anti-inflammatory $(14,15)$, antioxidant (16), anticancer (17) and anti-aging (18-22) effects as well as the promotion of hair growth $(23,24)$. PG contains many other ingredients such as sugars, proteins and lipids besides ginsenosides. Ginsenosides are a unique component of ginseng that is found only in ginseng, while sugars and proteins are common components of other plants. Also, various studies have indicated that the pharmacological effect of ginseng is derived from ginsenosides $(25,26)$. Recently, the authors reported that PG extract, which is a ginsenoside-enriched PG extract made using the repeated fractionalizing method, 
significantly enhanced the proliferation of hDPCs, potassium channel-opening activity, and human HF growth via a mechanism similar to that of minoxidil (27). Usually, ginsenosides of commercial PG extract are 3-6\%, but a ginsenoside-enriched PG extract are concentrated up to $20 \%$ using the preparation method used. The major ginsenosides detected in the ginsenoside-enriched PG extract were Rb1, Rb2, Rc, Rd, Re and Rg1. One of them, ginsenoside Re showed the highest level among the six ginsenosides and its content was approximately $6.23 \%(w / w)(27)$. In the current study, the authors investigated the inhibitory effect of ginsenoside-enriched PG extract on DKK-1-induced apoptosis in HFs in addition to the underlying mechanism of action.

\section{Materials and methods}

The preparation of PG extract. The authors conducted experiments using the same samples as PG extract, which had hair growth effect in our previous studies (27). The root of PG was obtained from Geumsan Ginseng Market (Geumsan-gun, Korea). The dried and crushed roots of PG (300 g) were extracted with $70 \%$ aqueous ethanol at $50^{\circ} \mathrm{C}$ for $8 \mathrm{~h}$. The extracts were filtered and concentrated under reduced pressure at $60{ }^{\circ} \mathrm{C}$. The residue was dissolved with $100 \%$ ethanol and repeat filtration and vacuum distillation.

Materials. Minoxidil, MTT and dimethyl sulfoxide were purchased from Sigma-Aldrich; Merck KGaA (Darmstadt, Germany). Human DKK-1 was purchased from R\&D Systems, Inc. (Minneapolis, MN, USA).

Isolation and cultures of human ORS keratinocytes. Non-balding scalp specimens were obtained from patients undergoing hair transplantation surgery (IRB:DKUH 2013-08-012-001). The medical ethical committee of the Dankook Medical College (Department of Dermatology, Cheonan, Korea) approved all of the described studies, and informed written consent was obtained from the patients. HFs were isolated and cultured by the previously described method, with minor modifications (28). Cultured ORS keratinocytes of early passage were used for the experiments and were maintained at $37^{\circ} \mathrm{C}$ in a humidified atmosphere with $5 \% \mathrm{CO}_{2}$.

MTT assay. Cell viability was determined using an MTT assay that was performed by a slight modification of the method described by Philpott et al (29). Briefly, ORS keratinocytes were seeded at a density of $2 \times 10^{4}$ cells/well into 96 -well plates and were cultured for $24 \mathrm{~h}$. Prior to treatment, the cells were cultured for $24 \mathrm{~h}$ in a growth supplement-free medium. The cells were then treated with PG extract and DKK-1 for $24 \mathrm{~h}$. The samples were assessed by measuring absorbance at $540 \mathrm{~nm}$ with a Synergy ${ }^{\mathrm{TM}} 2$ Multi-Detection Microplate Reader (BioTek Instruments, Inc., Winooski, VT, USA). The cell viability rates were calculated from the optical density readings and are represented as percentages of the control value (untreated cells).

Reverse transcription-quantitative polymerase chain reaction. The total RNA was isolated using TRIzol ${ }^{\mathrm{TM}}$ reagent (Invitrogen; Thermo Fisher Scientific, Inc., Waltham, MA,
USA), and $2 \mu \mathrm{g}$ RNA was reverse-transcribed into cDNA using SuperScript ${ }^{\circledR}$ III Reverse Transcriptase (Invitrogen; Thermo Fisher Scientific, Inc.). Quantitative real-time TaqMan PCR technology (TaqMan Universal PCR Master Mix, part no. 4304437) was used (Applied Biosystems; Thermo Fisher Scientific, Inc., Santa Clara, CA, USA). The cDNA samples were analyzed to determine the expression of the following: Hs00608023_m1 (Bcl-2), Hs00180269_m1 (Bax), and Hs02758991_g1 (GAPDH). Commercially available these probes were purchased and used in Thermo Fisher Scientific, Inc.

Terminal deoxynucleotidyl-transferase-mediated dUTP nick-end labelling (TUNEL) assay. A TUNEL kit (In Situ Cell Death Detection kit, Fluorescein, Roche Diagnostics GmbH, Mannheim, Germany) was used according to the manufacturer's protocol to evaluate apoptotic cells. Briefly, ORS keratinocytes at $2 \times 10^{4}$ cells/200 $\mu \mathrm{l}$ were seeded into eightchamber slides (Nunc Lab-Tek; Thermo Fisher Scientific, Inc., Roskilde, Denmark), were serum-starved for $24 \mathrm{~h}$, and were then treated with PG extract and DKK-1 for $24 \mathrm{~h}$. These cells were then fixed in $4 \%$ paraformaldehyde for $10 \mathrm{~min}$. After being washed with PBS, the cells were incubated with $0.1 \%$ Triton $\mathrm{X}-100$ in $0.1 \%$ sodium citrate for $1 \mathrm{~h}$ at room temperature. After washing, the cells were treated with the TUNEL reaction mixture and then were counterstained with 4',6-diamidino-2-phenylindole (DAPI) to visualize the nuclei. Representative images were taken with a fluorescence microscope (Olympus Corp., Tokyo, Japan) at x100 magnification.

Immunocytochemistry assay. ORS keratinocytes at $2 \times 10^{4}$ cells/200 $\mu \mathrm{l}$ were seeded into eight-chamber slides, and then treated with DKK-1 and PG extract for $24 \mathrm{~h}$. These cells were then fixed in $4 \%$ paraformaldehyde for $10 \mathrm{~min}$. After washing with Dulbecco's PBS, the cells were permeabilized with $0.1 \%$ Triton X-100 in PBS for 10 min at room temperature and then blocked with 5\% BSA in $0.05 \%$ Triton X-100 for $30 \mathrm{~min}$ at room temperature. The samples were incubated with Bcl-2 (1:200 dilution, sc-783; Santa Cruz Biotechnology, Inc., Dallas, TX, USA) and Bax antibody (1:200 dilution, sc-6236; Santa Cruz Biotechnology, Inc.) at $4^{\circ} \mathrm{C}$ overnight. They were then washed two times with PBS and four times with distilled water followed by incubation with a Alexa Fluor ${ }^{\mathrm{TM}} 488$ antirabbit (1:200 dilution, A-11034; Thermo Fisher Scientific, Inc.) and Texas Red ${ }^{\mathrm{TM}}-\mathrm{X}$ anti-rabbit (1:200 dilution, T-6391; Thermo Fisher Scientific, Inc.) secondary antibodies in 5\% BSA blocking solution for $2 \mathrm{~h}$ at room temperature. All samples were counterstained with DAPI to visualize the nuclei. Representative images were taken with a fluorescence microscope (Olympus Corp.) at x100 magnification.

HF organ culture and assessment of hair elongation. Anagen HFs from human scalp skin specimens were obtained from patients undergoing hair transplantation surgery. The medical ethical committee of the Dankook University Hospital (Cheonan, Korea) approved all of the described studies. A total of six HFs/well in 24-well plates were cultured in William's $\mathrm{E}$ medium at $37^{\circ} \mathrm{C}$ in a humidified atmosphere with $5 \% \mathrm{CO}_{2}$ in $500 \mu \mathrm{l}$ basal medium supplemented with $10 \mu \mathrm{g} / \mathrm{ml}$ insulin, $10 \mathrm{ng} / \mathrm{ml}$ hydrocortisone, $2 \mathrm{mM}$ L-glutamine, 0.1\% Fungizone, 


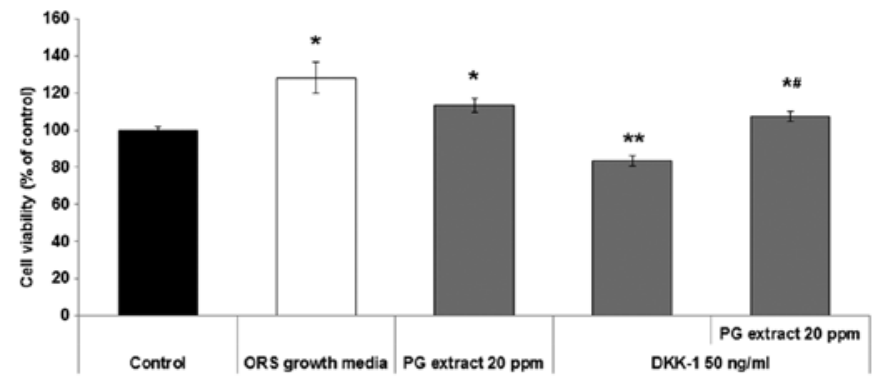

Figure 1. PG extract promotes ORS keratinocyte proliferation and abrogates DKK-1-mediated cell reduction. Cells were treated with $50 \mathrm{ng} / \mathrm{ml} \mathrm{DKK}-1$ or 20 ppm PG extract or with both DKK-1 and PG extract for 1 day, and an MTT assay was assessed on day 1 . ORS growth media served as a positive control for ORS keratinocyte promotion. Results were expressed as mean \pm standard deviation of percentage change compared to the control. Statistically significant differences were determined by t-test $\left({ }^{*} \mathrm{P}<0.05,{ }^{* *} \mathrm{P}<0.01 \mathrm{vs}\right.$. control; ${ }^{\#} \mathrm{P}<0.05$ vs. DKK-1-treated control). PG, Panax ginseng; ORS, outer root sheath; DKK-1, Dickkopf-1; TUNEL, terminal deoxynucleotidyl-transferasemediated dUTP nick-end labelling; DAPI, 4',6-diamidino-2-phenylindole.

$10 \mu \mathrm{g} / \mathrm{ml}$ streptomycin and $100 \mathrm{U} / \mathrm{ml}$ penicillin according to Philpott's method, as previous described (30). Each experimental group contained at least 30 anagen HFs derived from three different human donors/volunteers. DKK-1 $(50 \mathrm{ng} / \mathrm{ml})$ was determined to optimally induce catagen-like changes, shorten hair shaft length and hair bulbs with minimal other histological alterations to the HFs. Either the PG extract or MNX was added at the concentration, respectively, of $20 \mathrm{ppm}$ or $50 \mu \mathrm{M}$. The incubation medium was renewed every 2 days. The HF elongation was measured directly at 2,5 and 7 days of culture using a light stereo microscope (Olympus Corp.).

Statistical analysis. The results are expressed as mean \pm standard deviation. The data was analyzed using a Student's t-test, and the two-tailed value of $\mathrm{P}<0.05$ was considered to indicate a statistically significant difference. The data was processed by SPSS software for Windows, version 22.0 (SPSS Inc., Chicago, IL, USA).

\section{Results}

$P G$ extract stimulates proliferation and inhibits apoptosis in ORS keratinocytes. To investigate the potential role of PG extract on the proliferation and inhibition of apoptosis in ORS keratinocytes, the authors performed an MTT assay one day after treatment in the presence or absence of DKK-1 and PG extract. The PG extract and DKK-1 concentrations were determined by a previous study of the authors (data not shown). The results indicated that the $\mathrm{PG}$ extract enhanced the proliferation of ORS keratinocytes (Fig. 1) compared to untreated negative controls and enhanced ORS growth cultured positive controls with growth supplement medium. Treatment with DKK-1 (50 ng/ml) significantly inhibited the viability of ORS keratinocytes compared to the untreated negative control. PG extract significantly counteracted the inhibitory effect of apoptosis by DKK-1 on ORS keratinocyte (Fig. 1) viability.

To confirm the inhibitory effect of apoptosis by the PG extract in ORS keratinocytes, a TUNEL assay was performed. TUNEL-positive cells undergoing apoptosis significantly increased when ORS keratinocytes (Fig. 2A and B) were
Table I. Quantification of apoptosis in ORS keratinocytes by counting TUNEL-positive cells manually.

\begin{tabular}{lc} 
Sample & Apoptosis $(\%)$ \\
\hline Control & $7.92 \pm 5.50$ \\
DKK-1 $(50 \mathrm{ng} / \mathrm{ml})$ & $40.29 \pm 17.64^{\mathrm{a}}$ \\
PG extract $(20 \mathrm{ppm})+\mathrm{DKK}-1(50 \mathrm{ng} / \mathrm{ml})$ & $8.47 \pm 1.68^{\mathrm{b}}$ \\
$\operatorname{Rb} 1(1 \mu \mathrm{M})+\mathrm{DKK}-1(50 \mathrm{ng} / \mathrm{ml})$ & $33.15 \pm 10.34^{\mathrm{a}}$ \\
$\operatorname{Re}(1 \mu \mathrm{M})+\mathrm{DKK}-1(50 \mathrm{ng} / \mathrm{ml})$ & $32.30 \pm 13.01$ \\
$\operatorname{Rg} 1(1 \mu \mathrm{M})+\mathrm{DKK}-1(50 \mathrm{ng} / \mathrm{ml})$ & $18.05 \pm 1.54$
\end{tabular}

All values were expressed as mean \pm standard deviation. Statistically significant differences were determined by $\mathrm{t}$-test $\left({ }^{\mathrm{a}} \mathrm{P}<0.05\right.$ vs. control; ${ }^{\mathrm{b}} \mathrm{P}<0.05$ vs. DKK-1-treated control). TUNEL, terminal deoxynucleotidyl-transferase-mediated dUTP nick-end labelling; DKK-1, Dickkopf-1; PG, Panax ginseng; ORS, outer root sheath.

treated with $50 \mathrm{ng} / \mathrm{ml}$ of DKK-1. The PG extract decreased TUNEL-positive cells despite co-treatment with DKK-1.

Previously, it was found that there are many kinds of ginsenosides in total extract by high-performance liquid chromatography analysis (27). In order to determine which of these ginsenosides is most effective, we selected three representative ginsenosides of PG extract. (Rb1, Re, Rg1) and conducted further experiments. As a result (Fig. 2C and Table I), it was demonstrated that each ginsenoside inhibited apoptosis induced by DKK-1 to some extent. However, each effect was found to be insufficient for total extract.

$P G$ extract regulates the expression of apoptosis-related genes in ORS keratinocytes. To further investigate the relevance of the anti-apoptotic effects of PG extract on DKK-1, changes in the expression of apoptosis-related genes were examined by RT-qPCR. ORS keratinocytes were treated with PG extract and/or DKK-1 for $24 \mathrm{~h}$. DKK-1-induced apoptosis was accompanied by Bcl-2/Bax expression in many cells, including $\mathrm{HF}$ cells $(12,13)$. In ORS keratinocytes, DKK-1 treatment significantly decreased anti-apoptotic factor Bcl-2 expression. PG extract alone increased Bcl-2 expression four times more than the untreated control, and it reversed the DKK-1-induced inhibition of Bcl-2 expression. DKK-1 also induced the expression of the pro-apoptotic factor Bax. PG extract significantly inhibited Bax expression in ORS keratinocytes (Fig. 3A) despite the presence of DKK-1. In other words, PG extract promotes ORS keratinocyte survival and increases the ratio of Bcl-2/Bax to further inhibit the cell death (Fig. 3B). Increased protein level of Bcl-2 and decreased protein of Bax were confirmed by immunocytochemistry (Fig. 3C). These results indicated that the effect of PG extract was mediated through Bcl-2/Bax expression on ORS keratinocytes.

PG extract abrogates DKK-1 inhibition of hair shaft elongation in human HF organ culture. In order to examine the effect of PG extract in the presence or absence of DKK-1 at the organ level, the authors performed an ex vivo culture of whole human scalp HFs. Minoxidil (MNX) and vehicle served as positive and negative controls, respectively. HFs treated with PG extract 
A

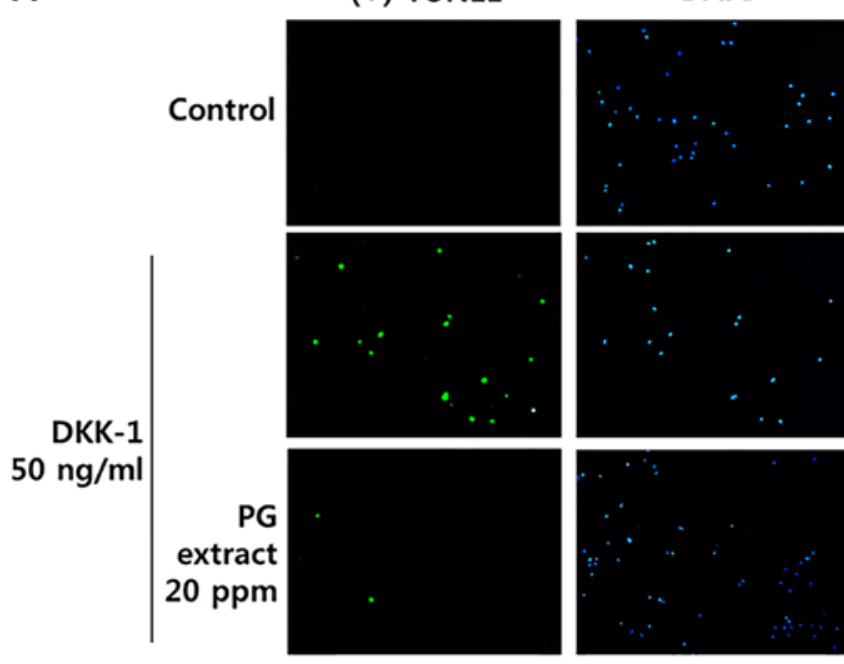

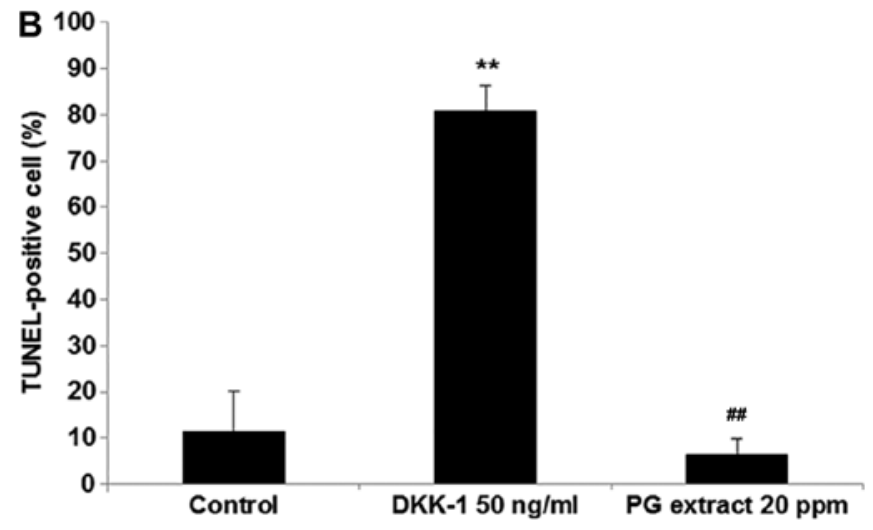

DKK-1 $50 \mathrm{ng} / \mathrm{ml}$

C

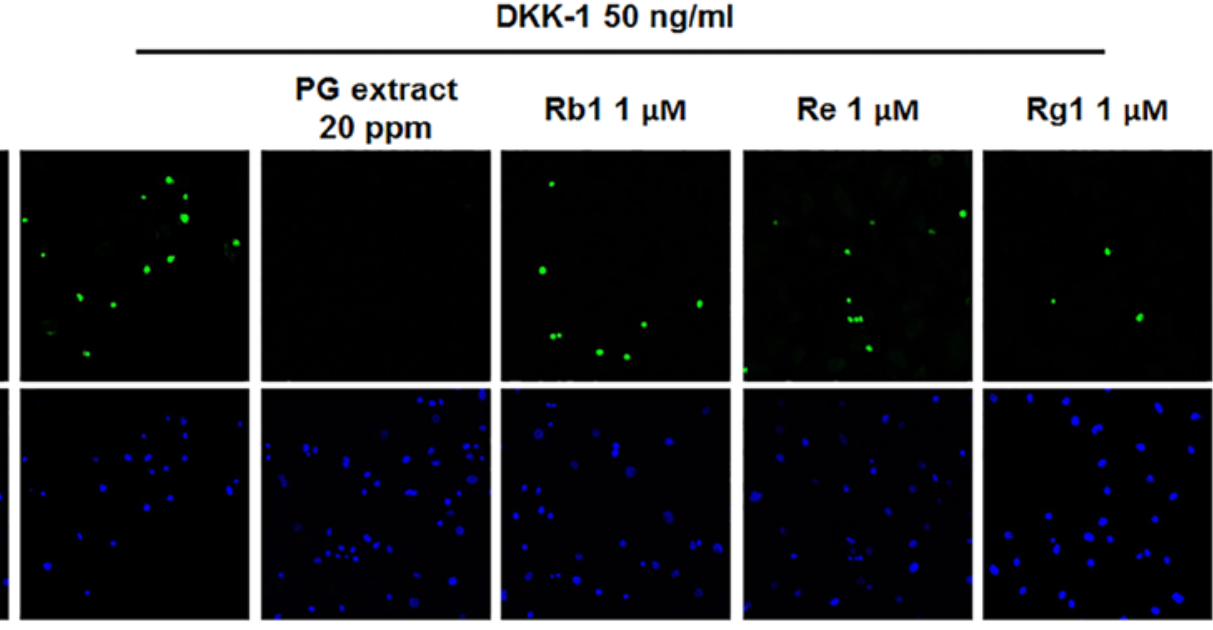

Figure 2. PG extract (A) and three representative ginsenosides of PG extract (C) inhibits cell death by apoptosis in DKK-1-treated ORS keratinocytes. Cells were treated with $50 \mathrm{ng} / \mathrm{ml}$ of DKK-1 or $20 \mathrm{ppm}$ PG extract or $1 \mu \mathrm{M}$ ginsenosides or with both DKK-1 and PG extract or ginsenosides for 1 day and were stained using the TUNEL method. Corresponding 4',6-diamidino-2-phenylindolenuclear staining (blue) is also shown at x100 magnification. TUNEL-positive apoptotic cells (green) were counted, and data are mean \pm standard deviation of percentage change compared to the control from three independent experiments (B). Statistically significant differences were determined by t-test $\left({ }^{* *} \mathrm{P}<0.01 \mathrm{vs}\right.$. control; ${ }^{\# \#} \mathrm{P}<0.01$ vs. DKK-1-treated control). PG, Panax ginseng; DKK-1, Dickkopf-1; ORS, outer root sheath; TUNEL, terminal deoxynucleotidyl-transferase-mediated dUTP nick-end labelling.

grew longer than the negative control HFs at 5 days, which was similar to the growth of HFs treated with MNX. This result is consistent with the authors' previous study (27). A low dose of DKK-1 $(<50 \mathrm{ng} / \mathrm{ml})$ produced no significant impairment of hair shaft elongation compared to the vehicle (data not shown), but a dose of $50 \mathrm{ng} / \mathrm{ml}$ DKK-1 significantly inhibited hair shaft elongation. The authors observed a narrower hair bulb in HFs treated with DKK-1 at the $50 \mathrm{ng} / \mathrm{ml}$ dose, which is reminiscent of catagen-like regressive changes. They also measured the anagen/catagen ratio (Fig. 4A), and DKK-1 treatment resulted in anagen-to-catagen changes in the HF organ culture. Thus, DKK-1 treatment at the dose of $50 \mathrm{ng} / \mathrm{ml}$ was used to establish an ex vivo model of HF catagen induction. With co-incubation of the PG extract and DKK-1, the PG extract significantly abrogated DKK-1-induced growth inhibition of cultured HFs ex vivo (Fig. 4B).

$P G$ extract regulates the expression of hair growth-related factors in HFs. There is evidence to suggest that several factors such as cytokine, growth factor, and apoptosis-related factor are involved in the hair growth cycle (31). In the above results, the authors already confirmed that PG extract regulates hair growth-related factors at the in vitro level. To confirm the inhibitory effect of apoptosis by PG extract at the ex vivo level, changes in the gene expression were examined by RT-qPCR using HFs treated with PG extract and/or DKK-1 for 2, 5 and 7 days. The DKK-1 treatment significantly decreased anti-apoptotic factor Bcl-2 expression at 2 days. At the same time point, the PG extract completely abolished the effect of DKK-1 on Bcl-2 expression in HFs. On the other hand, DKK-1 treatment significantly increased Bax expression in HFs, whereas the PG extract strongly inhibited this induction of Bax for 5- and 7-day HFs (Fig. 5). Of note, the PG extract affected only Bax expression and not Bcl-2 in longer ex vivo culture experiments. These results suggested that PG extract antagonizes DKK-1-induced catagen-like changes, in part, through the regulation of apoptosis-related factor expression in HFs. 

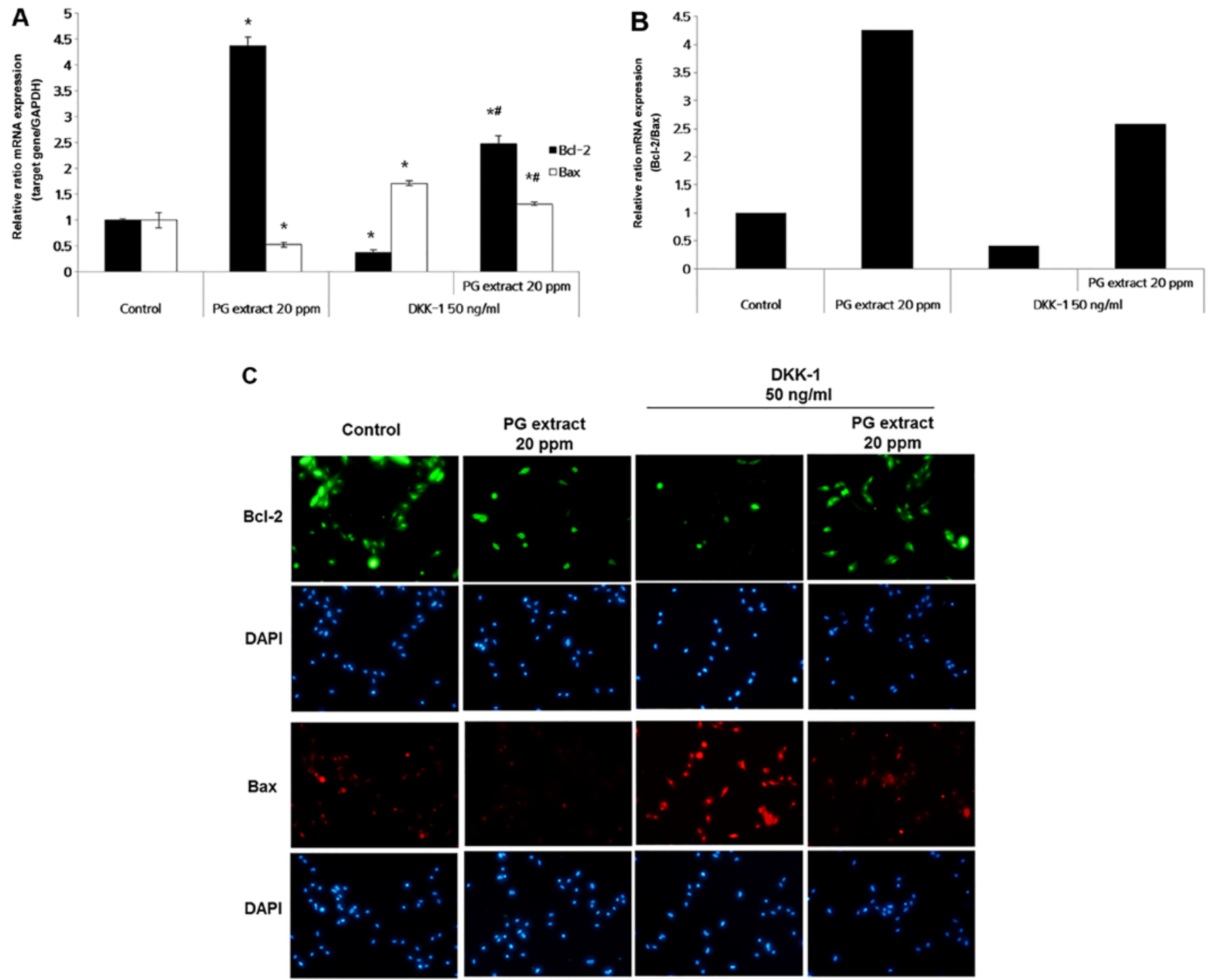

Figure 3. PG extract regulates the mRNA level expression of apoptosis-related genes in DKK-1-treated ORS keratinocytes (A) and their ratio (B). Cells were treated with $50 \mathrm{ng} / \mathrm{ml}$ DKK-1 or 20 ppm PG extract or with both DKK-1 and PG extract for 1 day. The levels of Bcl-2 and Bax were examined by RT-qPCR using a gene probe, respectively. Each relative mRNA level of Bcl-2 and Bax is presented as mean \pm standard deviation from three independent experiments. Statistically significant differences were determined by t-test. Expression of protein levels of Bcl-2 and Bax were confirmed by immunocytochemistry staining using specific anti-Bcl-2 and anti-Bax antibodies (green) and corresponding DAPI nuclear staining (blue) $(\mathrm{C})$. ( ${ }^{*} \mathrm{P}<0.05$ vs. control; ${ }^{*} \mathrm{P}<0.05$ vs. DKK-1-treated control). PG, Panax ginseng; DKK-1, Dickkopf-1; ORS, outer root sheath; DAPI, 4',6-diamidino-2-phenylindole.

\section{Discussion}

The major finding of the current study is that PG extract antagonizes DKK-1-induced HF changes, resulting in hair loss. Previous studies $(14-24,27)$ revealed that PG regulates a variety of biological effects such as anti-inflammatory, antioxidant, anticancer, and anti-aging effects, and of course, the promotion of hair growth. Recently, the authors prepared a highly concentrated ginseng extract with the repeated fractionalizing method and found that the PG extract contained $194.8 \mathrm{mg} / \mathrm{g}$ $(19.48 \% \mathrm{w} / \mathrm{w})$ of ginsenosides (27). Its ginsenoside content was $\sim 3$ times higher than that of commercial ginseng extracts for oral supplements in Korea and 14 times higher than that of conventional ginseng root extract (32). This newly prepared PG extract for treatment showed a significant hair growth effect with cultured hDPCs and HFs, which was comparable to the growth from minoxidil (27). Despite previous studies of PG and its effects associated with hair growth $(23,24)$, the mechanism underlying the apoptosis response, particularly the induction of DKK-1, has not been studied with respect to PG.

DKK-1 is well known as a WNT antagonist. It induces apoptosis and inhibits the proliferation of cancer cells $(33,34)$. DKK-1 is also inducible by dihydrotestosterone (DHT), and the level of DKK-1 is increased in the scalps of patients with male-pattern baldness compared to normal levels (11), suggesting that DKK-1 is involved in DHT-mediated balding in androgenic alopecia. It was also found that DKK-1 is highly expressed during the anagen-to-catagen transition. This implies that DKK-1 promotes the regression of HFs by blocking Wnt/ $\beta$-catenin signaling and by inducing apoptosis in follicular keratinocytes (13). The authors supposed that DKK-1 might inhibit hair growth and cell proliferation via apoptosis. As shown in these results, the viability of ORS keratinocytes was decreased by DKK-1. A TUNEL assay 

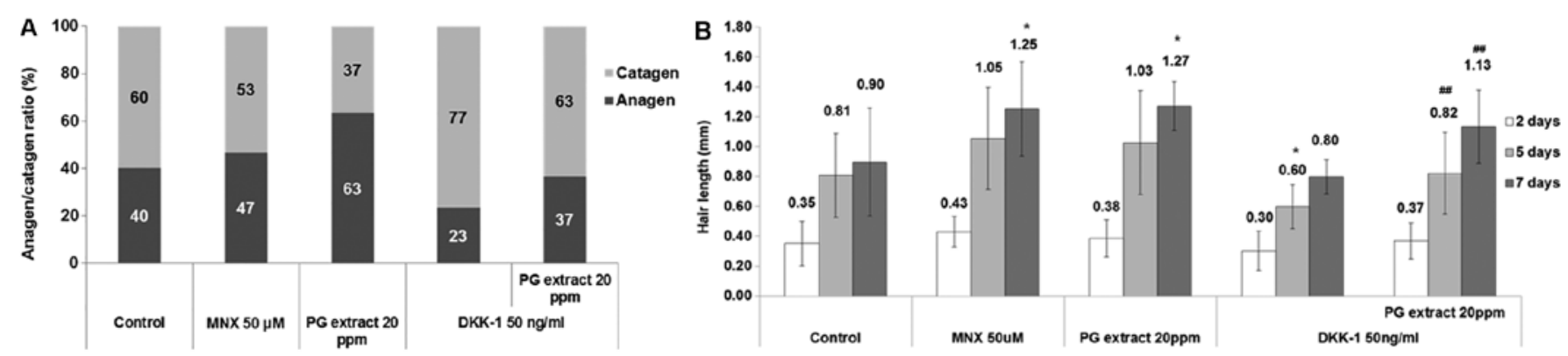

C DKK-1 $50 \mathrm{ng} / \mathrm{ml}$

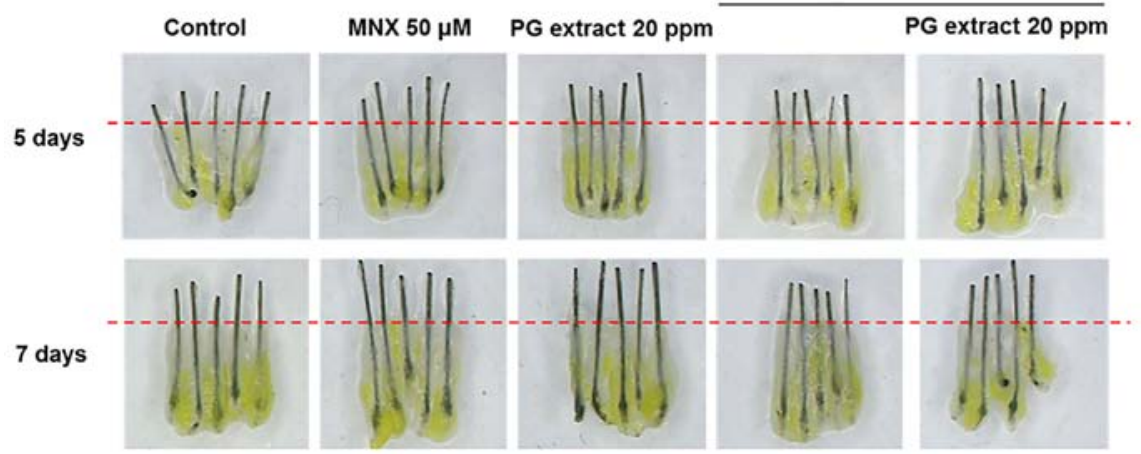

Figure 4. PG extract abrogates DKK-1 inhibition of hair growth in HF organ culture. A total of 150 human scalp hair follicles with intact dermal papillae were obtained and treated for 7 days with DKK-1 $(50 \mathrm{ng} / \mathrm{ml})$ or PG extract $(20 \mathrm{ppm})$ or with both DKK-1 and PG extract. The cycle of each hair follicle were presented on days 7 (A). The length of each hair follicle was measured under a microscope on days 2,5 and 7 . The relative length of each hair shaft is shown as mean \pm standard deviation (B). Images of representative hair follicles at days 5 and 7 are shown (C). MNX (50 $\mu \mathrm{M})$ served as the positive control for the stimulation of hair follicle growth. All values were expressed as mean \pm standard deviation. Statistically significant differences were determined by $\mathrm{t}$-test ( $\mathrm{P}<0.05$ vs. control; ${ }^{\# \#} \mathrm{P}<0.01$ vs. DKK-1-treated control). PG, Panax ginseng; DKK-1, Dickkopf-1; HFs, hair follicles; MNX, minoxidil.

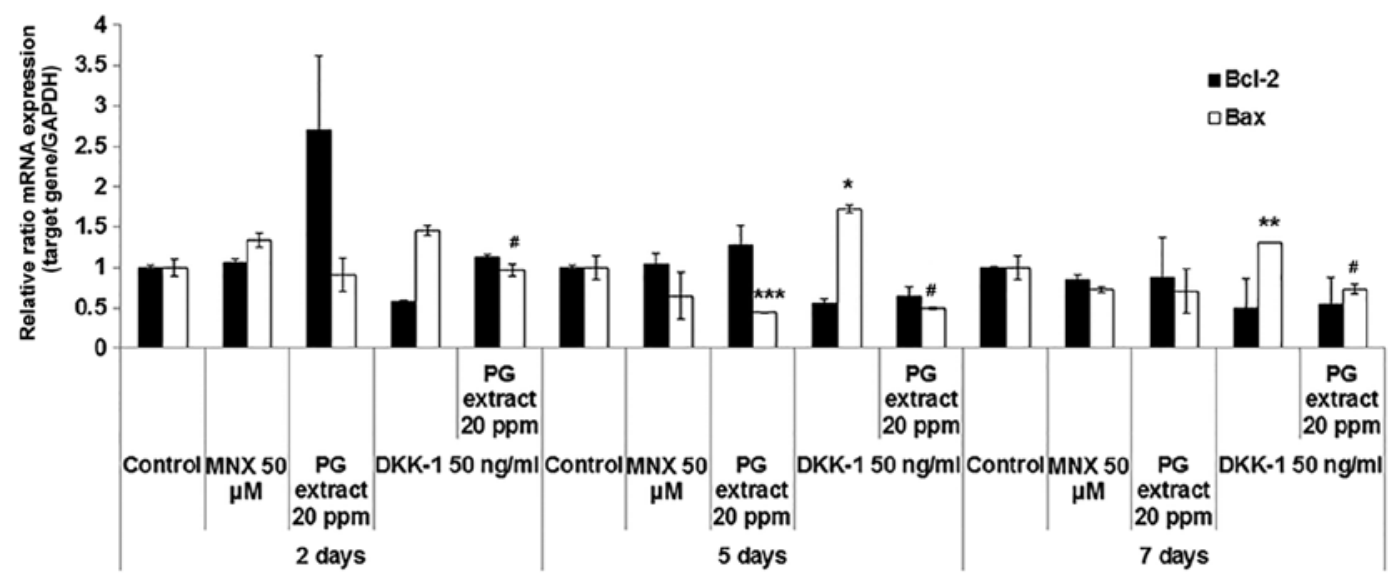

Figure 5. PG extract regulates the expression of apoptosis-related and hair cycle-related genes in DKK-1-treated HFs. HFs were treated with $50 \mathrm{ng} / \mathrm{ml}$ DKK-1 or 20 ppm PG extract or with both DKK-1 and PG extract for 2, 5 and 7 days. The levels of apoptosis-related genes (Bcl-2 and Bax) were examined by reverse transcription-quantitative polymerase chain reaction using a gene probe respectively. Each relative mRNA level of the target genes is shown as mean \pm standard deviation from three independent experiments. Statistically significant differences were determined by $\mathrm{t}$-test $\left({ }^{*} \mathrm{P}<0.05,{ }^{* * *} \mathrm{P}<0.01,{ }^{* * * *} \mathrm{P}<0.001 \mathrm{vs}\right.$. control; ${ }^{\#} \mathrm{P}<0.05$ vs. DKK-1-treated control). PG, Panax ginseng; DKK-1, Dickkopf-1; HFs, hair follicles; MNX, minoxidil.

demonstrated that the decreased cell viability was mediated by apoptosis.

During catagen, HFs undergo apoptosis, and there is a decline in the apoptotic protein Bcl-2 and an increase in the pro-apoptotic protein Bax (35). The ratio of these factors is important in regulating the hair cycle. In previous studies, DKK-1 treatment rapidly changed the anti-apoptotic protein Bcl-2. DKK-1 promoted the pro-apoptotic protein Bax in a dose-dependent manner $(12,13)$. Therefore, the present study investigated the effect of PG extract in ORS keratinocytes; the extract induces apoptosis by DKK-1 in these cells. The PG extract alone significantly increased cellular proliferation. It was correlated with the mRNA level of Bcl-2 expression increase, and it also inhibited Bax gene expression (Fig. 3A and B). Furthermore, when the PG extract was co-treated with DKK-1, the effect of the DKK-1 was inhibited. This suggested that PG 
extract may abolish the apoptotic signal stimulation of DKK-1 and help ORS keratinocytes to survive.

HF organ culture is now considered a useful tool for evaluating the effect of hair growth ex vivo. To further confirm the results shown in the above data, the authors investigated the effect of PG extract on DKK-1-induced catagen-like changes in cultured human HFs. It was shown that catagen-like morphological change was induced in DKK-1-treated HFs. Hair growth was also inhibited by DKK-1 during the incubation period. The PG extract significantly stimulated hair elongation, overcoming the inhibitory effect of DKK-1-induced hair growth, and finally, the hair was significantly more elongated than the untreated control.

In summary, PG extract has the potential to protect apoptosis in HFs. These findings suggested that PG extract may enhance ORS and hDPC stimulation of HF growth despite the presence of DKK-1, a strong catagen inducer via apoptosis.

\section{Acknowledgements}

The study was supported by grants from Amorepacific R\&D Center (R17E117001).

\section{References}

1. Inui S, Fukuzato Y, Nakajima T, Yoshikawa K and Itami S: Androgen-inducible TGF-betal from balding dermal papilla cells inhibits epithelial cell growth: A clue to understand paradoxical effects of androgen on human hair growth. FASEB J 16: 1967-1969, 2002.

2. Li L, Mignone J, Yang M, Matic M, Penman S, Enikolopov G and Hoffman RM: Nestin expression in hair follicle sheath progenitor cells. Proc Natl Acad Sci USA 100: 9958-9961, 2003.

3. Reynolds AJ and Jahoda CA: Cultured dermal papilla cells induce follicle formation and hair growth by transdifferentiation of an adult epidermis. Development 115: 587-593, 1992.

4. Millar SE: Molecular mechanisms regulating hair follicle development. J Invest Dermatol 118: 216-225, 2002.

5. Botchkarev VA and Paus R: Molecular biology of hair morphogenesis: Development and cycling. J Exp Zoolog B Mol Dev Evol 298: 164-180, 2003.

6. Botchkareva NV, Ahluwalia G and Shander D: Apoptosis in the hair follicle. J Invest Dermatol 126: 258-264, 2006.

7. Sawaya ME, Blume-Peytavi U, Mullins DL, Nusbaum BP, Whiting D, Nicholson DW, Lotocki G and Keane RW: Effects of finasteride on apoptosis and regulation of the human hair cycle. J Cutan Med Surg 6: 1-9, 2002.

8. El-Domyati M, Attia S, Saleh F, Bassyouni M, Barakat M and Abdel-Wahab H: Evaluation of apoptosis regulatory markers in androgenetic alopecia. J Cosmet Dermatol 9: 267-275, 2010.

9. Oltvai ZN, Milliman CL and Korsmeyer SJ: Bcl-2 heterodimerizes in vivo with a conserved homolog, Bax, that accelerates programmed cell death. Cell 74: 609-619, 1993.

10. Müller-Röver S, Rossiter H, Lindner G, Peters EM, Kupper TS and Paus R: Hair follicle apoptosis and Bcl-2. J Investig Dermatol Symp Proc 4: 272-277, 1999.

11. Kwack MH, Ahn JS, Kim MK, Kim JC and Sung YK: Preventable effect of L-threonate, an ascorbate metabolite, on androgendriven balding via repression of dihydrotestosterone-induced dickkopf-1 expression in human hair dermal papilla cells. BMB Rep 43: 688-692, 2010.

12. Kwack MH, Sung YK, Chung EJ, Im SU, Ahn JS, Kim MK and Kim JC: Dihydrotestosterone-inducible dickkopf 1 from balding dermal papilla cells causes apoptosis in follicular keratinocytes. J Invest Dermatol 128: 262-269, 2008.

13. Kwack MH, Kim MK, Kim JC and Sung YK: Dickkopf 1 promotes regression of hair follicles. J Invest Dermatol 132: 1554-1560, 2012.

14. Hu S, Concha C, Lin F and Persson Waller K: Adjuvant effect of ginseng extracts on the immune responses to immunisation against Staphylococcus aureus in dairy cattle. Vet Immunol Immunopathol 91: 29-37, 2003.
15. Na HS, Lim YJ, Yun YS, Kweon MN and Lee HC: Ginsan enhances humoral antibody response to orally delivered antigen. Immune Netw 10: 5-14, 2010.

16. Hong CE and Lyu SY: Anti-inflammatory and anti-oxidative effects of korean red ginseng extract in human keratinocytes. Immune Netw 11: 42-49, 2011.

17. Yun TK and Choi SY: Preventive effect of ginseng intake against various human cancers: A case-control study on 1987 pairs. Cancer Epidemiol Biomarkers Prev 4: 401-408, 1995.

18. Cho S, Won CH, Lee DH, Lee MJ, Lee S, So SH, Lee SK, Koo BS, Kim NM and Chung JH: Red ginseng root extract mixed with Torilus fructus and Corni fructus improves facial wrinkles and increases type I procollagen synthesis in human skin: A randomized, double-blind, placebo-controlled study. J Med Food 12: 1252-1259, 2009.

19. Kang TH, Park HM, Kim YB, Kim H, Kim N, Do JH, Kang C, Cho Y and Kim SY: Effects of red ginseng extract on UVB irradiation-induced skin aging in hairless mice. J Ethnopharmacol 123: 446-451, 2009.

20. Kim S, Kang BY, Cho SY, Sung DS, Chang HK, Yeom MH, Kim DH, Sim YC and Lee YS: Compound K induces expression of hyaluronan synthase 2 gene in transformed human keratinocytes and increases hyaluronan in hairless mouse skin. Biochem Biophys Res Commun 316: 348-355, 2004.

21. Kwok HH, Yue PY, Mak NK and Wong RN: Ginsenoside $\mathrm{Rb}(1)$ induces type I collagen expression through peroxisome proliferator-activated receptor-delta. Biochem Pharmacol 84: 532-539, 2012.

22. Lee J, Jung E, Lee J, Huh S, Kim J, Park M, So J, Ham Y, Jung K, Hyun CG, et al: Panax ginseng induces human Type I collagen synthesis through activation of Smad signaling. J Ethnopharmacol 109: 29-34, 2007.

23. Matsuda H, Yamazaki M, Asanuma Y and Kubo M: Promotion of hair growth by ginseng radix on cultured mouse vibrissal hair follicles. Phytother Res 17: 797-800, 2003.

24. Kim SH, Jeong KS, Ryu SY and Kim TH: Panax ginseng prevents apoptosis in hair follicles and accelerates recovery of hair medullary cells in irradiated mice. In Vivo 12: 219-222, 1998.

25. Attele AS, Wu JA and Yuan CS: Ginseng pharmacology: Multiple constituents and multiple actions. Biochem Pharmacol 58: 1685-1693, 1999.

26. Leung KW and Wong AS: Pharmacology of ginsenosides: A literature review. Chin Med 5: 20, 2010.

27. Kim SN, Kim S, Hong YD, Park H, Shin SH, Kim AR, Park BC, Shin SS, Park JS, Park M, et al: The ginsenosides of Panax ginseng promote hair growth via similar mechanism of minoxidil. J Dermatol Sci 77: 132-134, 2015.

28. Magerl M, Kauser S, Paus R and Tobin DJ: Simple and rapid method to isolate and culture follicular papillae from human scalp hair follicles. Exp Dermatol 11: 381-385, 2002.

29. Philpott MP, Sanders DA and Kealey T: Effects of insulin and insulin-like growth factors on cultured human hair follicles: IGF-I at physiologic concentrations is an important regulator of hair follicle growth in vitro. J Invest Dermatol 102: 857-861, 1994.

30. Philpott MP, Green MR and Kealey T: Human hair growth in vitro. J Cell Sci 97: 463-471, 1990.

31. Philpott MP, Sanders D, Westgate GE and Kealey T: Human hair growth in vitro: A model for the study of hair follicle biology. J Dermatol Sci 7 (Suppl): S55-S72, 1994.

32. Kim SN, Ha YW, Shin H, Son SH, Wu SJ and Kim YS: Simultaneous quantification of 14 ginsenosides in Panax ginseng C.A. Meyer (Korean red ginseng) by HPLC-ELSD and its application to quality control. J Pharm Biomed Anal 45: 164-170, 2007.

33. Hall CL, Zhang H, Baile S, Ljungman M, Kuhstoss S and Keller ET: p21CIP-1/WAF-1 induction is required to inhibit prostate cancer growth elicited by deficient expression of the Wnt inhibitor Dickkopf-1. Cancer Res 70: 9916-9926, 2010.

34. Hirata H, Hinoda Y, Nakajima K, Kawamoto K, Kikuno N, Ueno K, Yamamura S, Zaman MS, Khatri G, Chen Y, et al: Wnt antagonist DKK1 acts as a tumor suppressor gene that induces apoptosis and inhibits proliferation in human renal cell carcinoma. Int J Cancer 128: 1793-1803, 2011.

35. Lindner G, Botchkarev VA, Botchkareva NV, Ling G, van der Veen $C$ and Paus R: Analysis of apoptosis during hair follicle regression (catagen). Am J Pathol 151: 1601-1617, 1997. 